points, they will not be in conformity with the International Regulations for Preventing Collisions at Sea, or the German Inland Water Rules.

However, this should not prevent us from trying to find a solution to the problem. On the contrary, if it is possible to carry through any practical tests and if any of the tested signalling devices qualifies, the results should be examined closely as to whether they can serve the navigator as a new means of navigational aid and thereby increase the general safety at sea. As the Maritime Safety Committee has already thoroughly dealt with the question, any positive results should be reported to Imco and proposals amending Rule 28 of the International Regulations for Preventing Collisions at Sea should be submitted to that organization.

A proposed installation. The following system appears to offer the advantages sought. It consists of two additional lights:

(a) Above the second masthead-light (or if there is only one masthead-light, then above this one) at a vertical distance of $\mathrm{I} \cdot 83 \mathrm{~m}$. a second top light as a signal light.

(b) Above the stern light at a vertical distance of $\mathrm{I} .83 \mathrm{~m}$. a second stern light as a signal light.

On the navigation bridge (or control platform) a signal-giving device should be provided which continuously transmits the course signals as light signals to the signalling lights. The course signal is operated when the manourre begins and has to be switched off as soon as the ship is following its new course. Switching is hand-operated (as in a car).

A sternway manouvre can be automatically transmitted into the signal lights as 3 shorts by contact-breaking on the engine telegraph. On vessels, which are equipped with a manœuvre printer, the emission of a signal can be automatically registered at clock time. The location of the described signal lights refers to a reference direction of the keel-line and the position lights of the vessel. This principle has been applied to the location of the customs lamp on vessels navigating on the German waterways. This makes a confusion with other lights on board or with light identifications, which are sent from beacons or light buoys in pilotage waters, almost impossible.

\title{
REFERENCES
}

1 United States Coast Guard Proceedings, 24, 9. 1967.

2 This Journal, 19, 394.

3 Hansa No. $23,24 / 63$ No. $14 / 65$.

\section{A Navigational Glossary}

IN a pungent note published in the October 1966 Journal (Bad Language, Journal, 19, 523), Wing Commander E. W. Anderson drew attention to some of the confusion that had arisen through lack of a precise and accepted navigational terminology. He suggested that if a number of the more contentious terms were defined and accepted by the Institutes of Navigation, the use of 'bad language' 
might die out. It has now been agreed that the British Standards Institution should compile a navigational glossary and to set things going the Institute has formed, under Wing Commander Anderson, a sub-committee to try to sort out some of the terms over which ambiguity has arisen.

The definitions given here have been agreed between the sub-committee for submission to the British Standards Committee. Obviously neither the British Standards Institution nor the Institute is yet committed to them.

Terms have been omitted which are:

(a) Adequately described in the Oxford Dictionary.

(b) Accepted in disciplines outside navigation.

(c) Obsolete, or are

(d) Amplifications or descriptions of techniques or equipment.

Where a term is obsolescent or its use is not recommended it has not been defined but has been referred to a preferred term. A term is defined without reference to another defined term unless the wording is considerably increased by this policy. In particular, definition by reference to two or more other terms has been avoided. In all wordings, any general description comes before a precise definition. Units are not included. In some cases the conventions suggested conflict with those used in this Journal.

\section{A. ORIENTATION}

1. AвEAM

2. Aileron

3. Attitude (At.)

4. BEAM

5. BeAm axis

6. Elevator

7. Elevon

8. FIN

9. FOR

10. Green

I1. HeEl

12. Horizontal

13. Lateral axis

14. LisT

15. Longitudinal AXIS

16. NORMAL AXIS
In the direction of the transverse axis or in the opposite direction.

An aerofoil surface in the wing of an aircraft used for control of roll.

The angle of the normal axis relative to the vertical, generally resolved into pitch or trim and roll or heel components.

The direction of the transverse axis or the opposite direction.

See Transverse axis.

A surface used to control a craft in pitch.

An aerofoil surface in the wing of an aircraft used for control of pitch and roll.

A surface used to stabilize a craft in any axis.

See Longitudinal axis.

The colour associated with starboard.

The short-term attitude in roll of a marine craft.

The plane at right angles to the direction of gravity modified by the acceleration set up by the rotation of the planet.

See Transverse axis.

The long-term attitude in roll of a marine vessel.

Generally along the main body of the craft in the direction of designed motion. Defined by the constructor. Perpendicular to the longitudinal axis and generally in the plane of symmetry. Orientated from the roof of the crew compartment towards the floor or as defined by the constructor. 


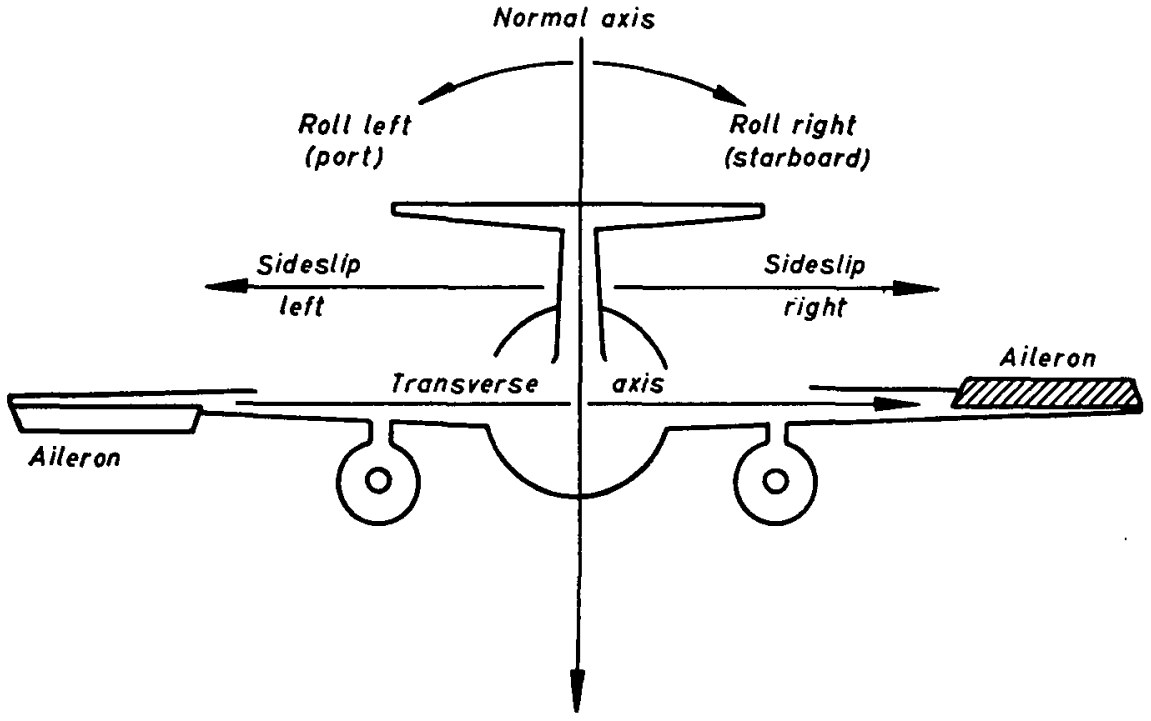

PIG. 1. View along longitudinal axis (from aft). Ailerons set to roll aircraft starboard (right)

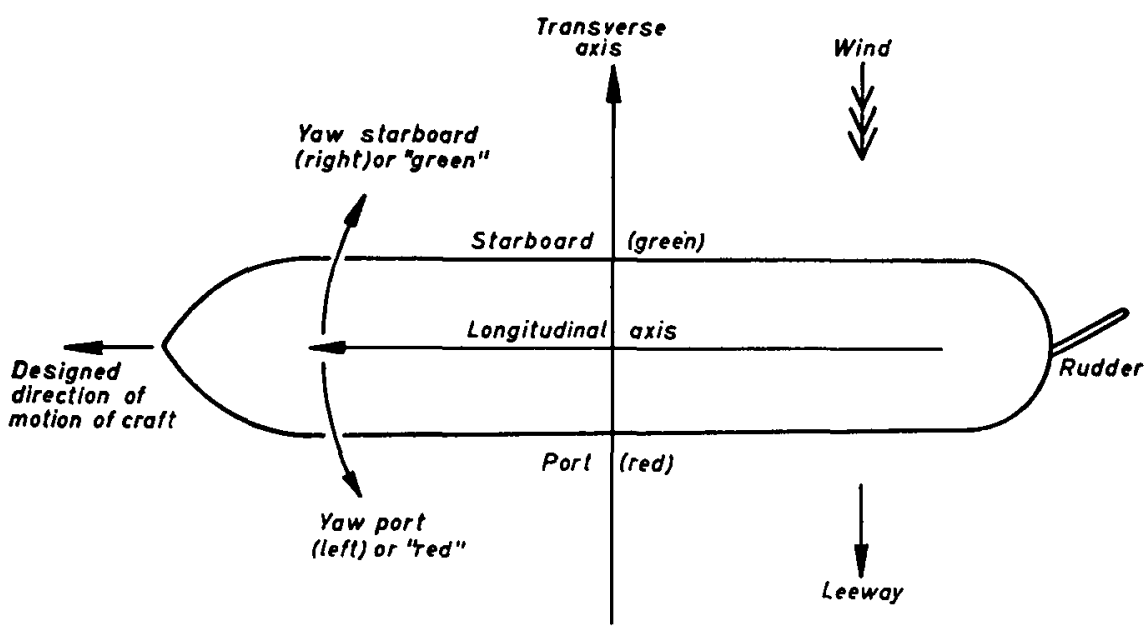

FIG. 2. View along normal axis (from above). Rudder set to yaw the ship starboard (right) 
17. PIrch

18. Pirching Down

19. Pitching UP

20. PORT

21. ReD

22. Roll

23. ROLLING LEFT

24. ROLLING RIGHT

25. RUDDER

26. StaRboard (STBD)

27. TAILeron

28. TRANSVERSE AXIS

29. TRIM

30. Vertical

31. YAW

32. YAWING LEFT OR PORT

33. YAWING RIGHT OR STARBOARD
Rotation about the transverse axis; named positive if it appears clockwise when viewed in the direction of this axis.

Negative pitch, i.e. bows or nose moving downwards. Positive pitch, i.e. bows or nose moving upwards.

In the direction opposite to the transverse axis. If a craft only travels forward, the word 'left' is commonly used instead.

The colour associated with port

Rotation about the longitudinal axis and named positive when it appears clockwise if viewed in the direction of this axis.

Negative roll, i.e. anti-clockwise looking ahead.

Positive roll, i.e. clockwise looking ahead.

A surface used to control a craft in yaw.

In the direction of the transverse axis. If a craft only travels forward, the word 'right' is commonly used instead.

Rear aerofoil surface used to control an aircraft in pitch and roll.

Perpendicular to the longitudinal axis and generally perpendicular to the plane of symmetry. Orientated to the right looking forward, that is $90^{\circ}$ anti-clockwise compared to the normal axis when viewed in the direction of the longitudinal axis.

The long-term attitude of a craft. In the instance of marine vessels, trim is confined to the pitch plane.

The direction of gravity (including the acceleration set up by the rotation of the planet).

Rotation about the normal axis and named positive when it appears clockwise if viewed in the direction of this axis.

Negative yaw, i.e. bows or nose moving to left.

Positive yaw, i.e. bows or nose moving to the right.

\section{B. DIRECTION, GENERAL}

1. Azimuth

Direction measured in the horizontal plane.

2. Astro-compass

An instrument or equipment that indicates direction in the horizontal plane from astronomical information.

3. Compass (Comp.) An instrument or equipment that measures direction in the horizontal plane.

4. Conversion The difference in direction between a rhumb line beANGLE tween two points and the initial or final direction of a great circle between those two points. 


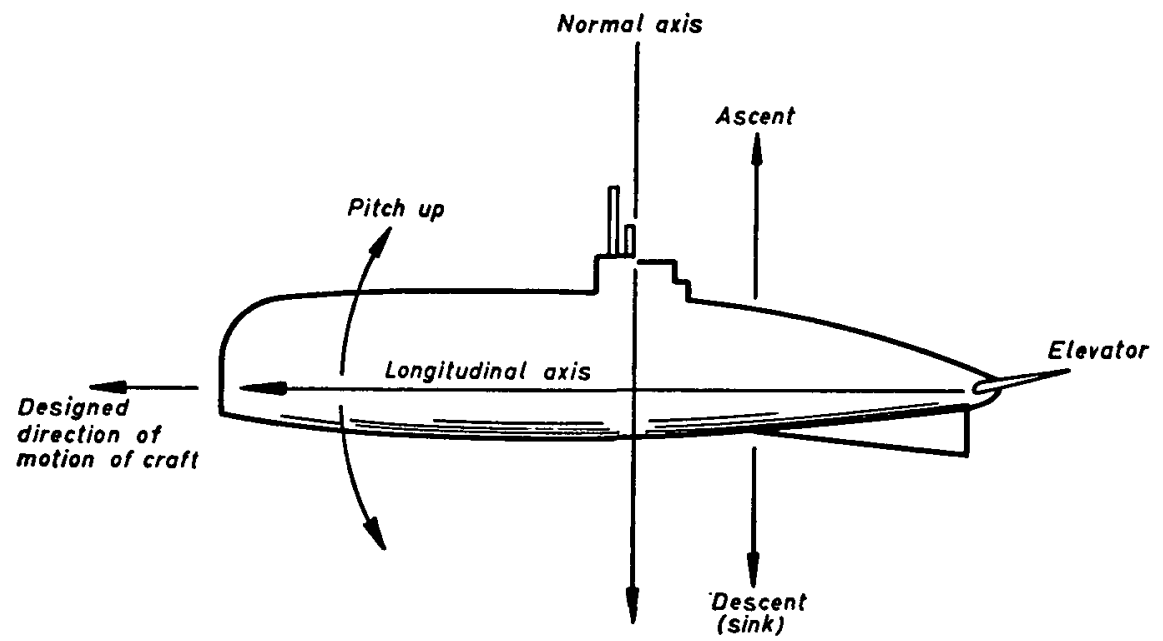

FG. 3. View along transverse axis (to starboard, right). Elevator set to pitch submarine up

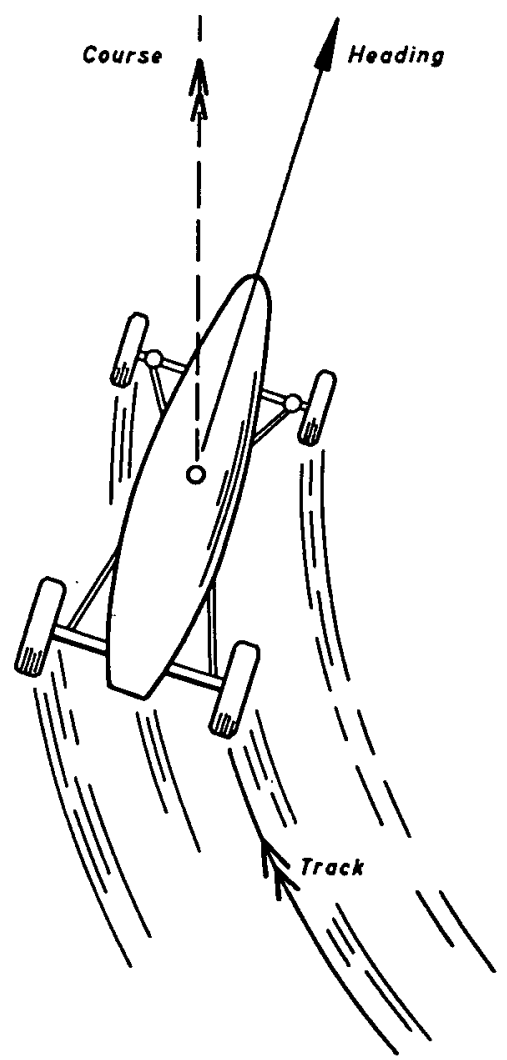

FIG. 4. Course, heading and track 
5. Deviation (Dev.) An angular deflection. In particular the deflection of the direction indicated by the magnetic compass compared to the magnetic meridian.

6. Directional A gyroscope used to maintain a heading reference preGYRO (DG) viously inserted by a compass.

7. Great Circle The shortest distance between two points on the surface of a sphere defined as the intersection of the surface with a plane passing through the two points and the centre of the sphere. A planet may be represented as a sphere.

8. Greenwich Grid A grid reference used in polar regions based on the Greenwich meridian.

9. GRID

A grid of directional lines drawn parallel to a given great circle on a specified map projection. (See also Section D, Item I 8.)

I0. GRID DIRECTION Direction measured clockwise through $360^{\circ}$ from the (G)

i I. Grivation

12. GYRO-COMPASS grid and suffixed $\mathrm{G}$.

The direction of the magnetic meridian measured clockwise through $360^{\circ}$ from the grid direction.

13. Gyro-Magnetic

An instrument or equipment that indicates direction by sensing the rotation of the supporting planet. An instrument or equipment the indications of direction derived from magnetic detectors.

14. Heading

15. Magnetic COMPASS

The direction of the longitudinal axis of the craft measured in the horizontal plane.

An instrument or equipment that indicates direction by sensing the magnetic field of the supporting planet.

I6. MAgnetic MERIDIAN

At any point, the direction of that part of the horizontal component of the magnetic field of a planet which runs towards the north magnetic pole.

17. Magnetic Direction measured clockwise through $360^{\circ}$ from the DIRECTION (M) magnetic meridian and suffixed $M$.

I8. Meridian

A great semi-circle joining the true-north and the truesouth poles.

19. Relative (R) Direction in the horizontal plane measured from the longitudinal axis of a craft either clockwise through $360^{\circ}$ and suffixed $R$ or to right or left of heading and named starboard (red) or port (green).

20. Rhumb LiNe A line on the surface of a planet which has a constant true direction.

21. True (T) Direction measured clockwise through $360^{\circ}$ from the direction of true north and suffixed $\mathrm{T}$.

22. True NORTH The great circle from a point joining it to the truenorth pole.

23. Variation (Varn.) The direction of the magnetic meridian measured in degrees east or west from true north. 


\section{VELOCITY}

1. AIr SPEED (AS) A measure of air pressure set up by the motion of the craft. The air pressure is expressed in terms of the speed needed to achieve that pressure under certain specified conditions.

2. Calibrated air Identical with rectified air speed. SPEED

3. Симв

4. Corrected air SPEED

5. Coriolis

6. Course (Co.)

7. Course MADE GOOD

8. Crab

9. Cross track

Io. Decrab

II. Dive

12. DRIFT

13. Equivalent air SPEED (EAS)

14. GROUND SPEED (GS)

15. INDICATED AIR SPEED (IAS)

16. INTENDED TRACK

17. KICK-OFF DRIFT (KOD)
The angle of the path relative to the horizontal when a craft is ascending in a pitched-up attitude.

Identical with rectified air speed.

On a rotating planet, the horizontal acceleration at right angles to a great circle necessary to enable a moving body to follow that great circle.

The direction of intended motion of a craft in the horizontal plane measured relative to the water in the instance of a waterborne craft but relative to the solid earth in all other craft.

The mean course actually achieved over a past period of time.

The deflection of the heading of a craft from its course. Measured in degrees to left or right of the course.

At right angles to track.

Removal of crab or drift prior to landing by the introduction of slideslip.

The angle of the path relative to the horizontal when a craft is descending in a pitched-down attitude.

Generally, a small unwanted movement. In particular, the motion of the water in the instance of a waterborne craft or the sideways motion of the air in the instance of an airborne craft. The consequent deflection of the direction of motion of the craft from its heading is known as Drift Angle, the word 'angle' being omitted when angular measurement is implied. Drift angle is measured in degrees to left or right of heading.

A measure of lift derived from rectified air speed corrected for compressibility.

The speed of a craft relative to the solid surface of the planet below it.

The uncorrected reading of pressure due to the speed of the craft through the air expressed in terms of the speed needed to achieve that pressure in a standard atmosphere at sea-level.

See Track.

Identical with decrab. 
18. LEEWAY

19. LoG

20. MACH Number (M) Speed through the air compared to the speed of sound.

2 I. Pitot

22. RATE OF CLIMB

23. Rate of DIVE

24. RATE OF TURN

25. RECTIFIED AIR SPEED (RAS)

26. Relative course, Course, speed, track or velocity measured relative to SPEED, TRACK a moving object, in particular a moving craft. OR VELOCITY

27. SET (OF CURRENT) The direction of motion of a current or tidal stream.

28. SIDESLIP

29. SINK

30. Static

3I. Track (Tr.)

32. TRACK MADE GOOD (TMG)

33. TRACK REQuired

34. True $(T)$

35. TRUE AIR SPEED (TAS)

36. TRUE NORTH

37. VeLOCITY

38. VSI

39. WATER SPEED
Speed along the transverse axis of a waterborne craft due to wind. The consequent deflection of the direction of motion of a craft is the leeway angle, the word 'angle' being omitted when angular measurement is implied. Leeway angle is measured to starboard or to port of heading.

An instrument that records speed through water. (See also Section D, Items $I_{3}$ and 17 .)

Pressure set up by motion through water or air. The pressure is detected by a pitot head and may be transmitted along a pitot tube.

Vertical speed in a climb.

Vertical speed in a dive.

A numerical value equal to the number of turns through $180^{\circ}$ achieved in one minute, i.e. Rate $\frac{1}{2}=90^{\circ} / \mathrm{min}$.

Indicated air speed corrected for installation and positioning errors.

Speed along the transverse axis of an airborne craft. The resultant deflection of the direction of forward motion is known as sideslip angle and is measured in degrees left or right from the heading.

Vertical descent not associated with any particular attitude.

Pressure of the air or water around a vehicle undisturbed by the vehicle motion. The pressure is detected by a static vent and may be transmitted along a static tube. The past path of the craft over the solid surface of the Earth. The mean direction of this path is known as the Track Angle, the word 'angle' being omitted when the sense is obvious.

A planned future track is known as an intended or required track.

Identical with Track.

See Track.

Direction measured clockwise through $360^{\circ}$ from the direction of true north and suffixed $T$.

The speed of a craft relative to the air around it.

The great circle from a point joining it to the true-north pole.

The combination of direction of motion and speed.

Vertical speed indicator

The speed of a marine craft relative to the water. 


\section{NA VIGATIONAL GENERAL}

1. Altitude (Alt.) The reading in feet of a pressure altimeter.

2. Bearing (Brng.) The direction of one object from another measured in the horizontal plane either in true, grid, magnetic or relative directions and suffixed T, G, M or R accordingly.

3. Bearing plate A device used to measure bearings visually from a craft.

4. CAPTURE The acquisition of a track guide by the control system of a craft.

5. Circular error A circle drawn around a point on a map or a chart to represent an error in position.

6. COCKED hat An area contained by three or more position lines.

7. Coupling The linking to a track guide of the control system of a craft.

8. Critical point The point beyond which the fuel required to complete a journey is less than the fuel required to return.

9. Curve of pursuit The path resulting from heading towards a target.

10. Curve of equal The line to a radio station at any point along which the BEARING true bearing of the station is constant.

II. CuT The angle between two position lines which are being used to establish the position of a craft.

12. Dead reckoning The calculation of position or course from track or (DR) ground speed or, in the instance of waterborne craft, from a past knowledge of course made good and water speed.

13. DECK LOG

A record that includes the navigational progress of a marine craft.

14. DEPTH

15. ETA

The depth of water below a stated datum.

16. FIX Estimated time of arrival.

A geographical position established by the intersection of two or more position lines.

17. Flight Log

A record that includes the navigational progress of an aircraft.

18. GRID, cIvil OR A pattern of squares of a constant size, drawn on a map MILITARY or chart of a given projection and covering a certain area, and used to define position. (See also Section B, Item 9.)

19. Height

The vertical distance above a stated datum.

20. HOLD

The control of a craft to ensure a constant height or depth.

21. Homing Navigation to a point by heading towards it.

22. LEG An interval of a planned journey along which course need not be changed.

23. Line of position See Position line.

24. LINE OF SHOOT The general direction in which a pattern of stations provides the greatest fixing accuracy.

25. LOCK OR LOCK-ON The control of a craft to ensure a constant speed or to follow a selected track.

26. Navigation Lights on a craft designed to show its motion and aspect LIGHTS at night. 
27. NORTHERLY The errors in a magnetic compass which are a maximum TURNING ERROR when the craft is headed true north or true south, caused by accelerations in turns.

28. PLOT To draw courses or tracks on a map or chart.

29. Pin POINT A geographical position established from visual information.

30. Point of No RETURN

31. Positioning The point on a journey beyond which a craft has insufficient fuel to return to its starting point.

An index error in a pressure instrument depending on ERROR the position on the outside of the craft where the pressure is detected.

32. Position error See Positioning error.

33. Position IINE A line along which the position of a craft has been established.

34. Proportional The altering of course in proportion to the rate of NAvigation change of bearing of an object in order to achieve interception.

35. Quickening The provision of early indications of action from rate information.

36. Pelorus See Bearing plate.

37. RANGE

A limiting distance or a distance which has been measured directly.

38. Run The distance travelled in a certain time.

39. RunNing FIX A fix obtained from successive position lines.

40. TRACK GUIDE

A navigation system that defines an intended track. (See Section C, Item 16.)

41. Transit position A position line produced when two external objects are LINE in line as viewed from the craft.

\title{
'The Schuler Pendulum and Inertial Navigation'
}

\author{
J. A. Lee
}

Mr. Bell and Professor Stratton have in the October Journal shed interesting light on the nature of inertial navigation as applied to navigation around a planet. It is a pity that $\mathrm{Mr}$. Bell has repeated that in such inertial systems vehicle position and velocity are deduced from measurement of acceleration. This idea is certainly misleading so far as understanding error propagation in these systems is concerned, if not actually wrong.

It is a fact that in a typical aircraft inertial navigator the output signal from a nominally horizontally stabilized accelerometer is fed to two successive integrators, but this is neither the whole nor the most essential part of the story except in systems used for comparatively short periods of time, viz. short compared with 84 minutes for systems used on our planet. 